\title{
THE ELECTRON MICROSCOPIC OBSERVATIONS OF TOBACCO MIOSAIC VIRUS-INFECTED TISSUE BY ULTRA-THIN SECTIONING *
}

\author{
CHIAIT MATSUI ** \\ [Received May 17, 1956]
}

The most powerfull method to observe the localization and morphology of the developing virus particles within host cells is a ultra-thin sectioning of the diseased tissues. However the application of this interest technique for the investigations on higher plant virology has been less pronounced.

In 1950, Black, Morgan and Wyckoff published the first electron micrograph of tobacco mosaic virus rod particles within host cell (1). Their observations suggesting the definite relationship between virus rod particles and chloroplasts, have been supported by the successive investigators, and it has been widely accepted that the chloroplast is a site of reduplication of some plant viruses $(6,10,11)$.

Since these investigations were undertaken at a time when the technique of ultra-thin sectioning was not so advanced, it seems likely to be extended for a more effective approach to this problem by using a newly developing technique.

The author's sincere thanks are due to Prof. Drs. T. Hirai, N. Higashi and J. Hidaka for their excellent discussions and kind supervisions offered to this subject. The Author is very much indebted to Mrs. T. Shimomura and A. Yamaguchi for the facilities up to the disposal, and also express harty thanks to Mr. H. Yotsumoto for taking the electron micrographs.

\section{MATERIALS AND METHODS}

Both tobacco mosaic diseased and healthy tobacco young leaves (Nicotiana tabacum L. var. Xanthi) were cut into pieces of some millimeter thick. The cut leaves were fixed by Leyon's fixation method: 0.5 per cent osmium tetraoxide at pH 7.0 for $1 \mathrm{hr}$. in a cold room at $0^{\circ} \mathrm{C}$, washed in running tap water for 2 hrs.s 0.5 per cent formaldehyde for $1 / 2 \mathrm{hr}$, running tap water for $1 \mathrm{hr}$., 0.3 per cent chromic acid for $1 \mathrm{hr}$, and running tap water for 2 hrs (7). The procedure was followed by ethanol-dehydration and n-butyl methacrylate-embedding (3). The ultrathin sections were prepared using a thermal expansion ultra-microtome with a glass knife (JUM Model 3, Japan Electron Optics Laboratory), A JEM Model 5 electron microscope (Japan Electron Optics Laboratory) was used at a potential of $80 \mathrm{KV}$. Although, in the previous investigations $(1,2,9,10,11)$, the methacrylic resins were removed with amyl acetate etc., this treatment seems not to provide any detailed information on the localization of specific compounds in the ultra-thin structures of fixed tissue. Accordingly, in the present study, all electron micrographs

\footnotetext{
* This study was technically supported by Japan Electron Optics Laboratory Co., Ltd.

** Laboratory of Plant Pathology (Director: Prof. T. Hirai), Faculty of Agriculture, Nagoya University. Anjyo, Aichi-ken, Japan.
} 
were taken without dissolving the methacrylate.

\section{RESULTS}

The chloroplasts in healthy tissue. The chloroplasts of healthy tobacco leaves are shown in Fig. 1. The chloroplasts are built up of the regularly organized grana embedded in stroma, and the grana consist of regularly arranged thin lamellae. These observations correspond to the ultra-thin structures of chloroplasts of some other higher plants $(7,13)$.

The vinusinfected parenchyma. The chloroplasts of virusminfected leaves are shown in Figs. 2 and 3. The chloroplasts are undergone the various grades of degeneration. We can easily observe the disintegration of regularly organized grana and the granulation of chloroplasts. These histological changes seemed to be taken as an indication to the yellow chlorotic symptoms of the infected leaves. Virus rod particles associated with disintegrated chloroplasts are shown in Figs. 4, 5, 6 and 7. In these electron micrographs, the masses of parallely arranged virus rod particles are found on the surface of chloroplasts, and not within the disintegrated chloroplasts. Furthermore the masses of virus rod particles without any association with chloroplasts could be observed near the cell walls. Frequently the fragments of membraneous substance were adhered to the masses of virus rod particles. The nature of this substance remains unknown.

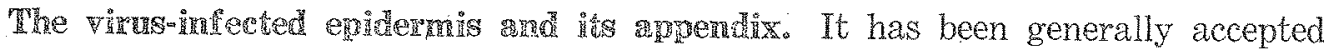
that the epidermis lacks the chloroplasts. In Fig. 9, virus rod particles are obviously produced. This observations are in excellent agreement with the previous investigations $(4,12,14)$. Fig. 10 represents the electron micrographs of the cross section of leaf epideral hair. These cells contain no chloroplasts, while the masses of virus rod particles are also found to be scattered all over there.

\section{DISCUSSION}

The idea that chloroplasts are the sites of virus duplication has already appeared. Since Kausche and Ruska published an electron micrograph showing filam entous particles protruding from chloroplast iragment of the tobacco mosaic virusinfected plant (5), the speculation that tobacco mosaic virus is duplicated in chlom roplasts and released from them into cytoplasm, is supported by some investigators with various electron micrographs $(2,6,9,10,11)$. However it seemed profitable to recall the fact that the association of virus rod particles with the disintegrated chloroplasts does not necessarily provide the direct proof of the duplication of virus within them. We must, in turn, consider what kind of association would be involved in there. Although, in the present study, we could also observe the masses of virus rod particles associating with disintegrated chloroplasts, the possibility that the association observed in the electron micrographs may be obtained as the result of a second hand attachment of virus rod particles duplicated in the other part of cell on to chloroplasts can not be negligible. Luria has assumed that the intimate association of virus rod particles with chloroplasts was due to a secondary invasion 
of these bodies (8).

In general, it has become increasingly clear that the grana in chloroplasts contain chromoprotein and some of the enzymatic activities. From these reasons, several attempts to explain that the protein and enzymatic activities in grana are utilized in the synthesis of new virus nucleoprotein through the sequence of various reactions may be possibly given. Nevertheless, these assumptions would not eventually shed considerable light toward the observations that the virus rod particles are developing associated with chloroplasts shown in the electron micrographs.

Since we can not find virus rod particles or virus-like particles within the disintegrated chloroplasts, even if one assumes that virus is reproduced in the chloroplasts and is released from them to the surrounding cytoplasm, it may be a safe assumption to say that the reproduction of virus in chloroplasts is an eclipse stage or vegetative phase of virus synthesis. So far as these electron micrographs reveal, however, it will be still open to discussion to decide, with the full realization, whether the definite association of virus rod particles with chloroplasts represents an actual virus duplication within them or it is merely due to a second hand association.

\section{SUMMARY}

Tobacco mosaic virus-infected tobacco leaf tissues were observed under the electron microscope coupled with the ultra-thin sectioning technique.

The masses of virus rod particles were observed in the diseased parenchyma, epidermis and its appendix. In the parenchyma, the chloroplasts of virus infected tissue were disintegrated and frequently the masses of parallely arranged virus rod particles were demonstrated associating with them. Sometimes the masses of virus rod particles without chloroplasts were found near the cell walls. In the present observations, virus rod particles or virus-like particles could not be found within disintegrated chloroplasts. With the aid of these findings the duplication of virus the in chloroplast was briefly discussed.

\section{References}

1) Black, L. M., Morgan, C. and Wyckoff, R. W. G.: Pro. Soc. Expt. Biol. and Med., 73, 119, 1950.

2) Hidaka, J. and Murano, H.: The annual meeting of the Japan Electron Microscope Soc., 1953.

3) Higashi, N.: Virus (Japan), 6, 69, 1956.

4) Holmes, F. O.: Phytopathology, 24, 1125, 1934.

5) Kausche, G. A. and Ruska, H.: Naturwissenschaften, 28, 303, 194.0.

6) Leyon, H.: Expt. Cell Research, 4, 362, 1953.

7) Leyon, H.: Expt. Cell Research, 7, 265, 1955.

8) Luria, S. E.: General Virology (1953), John Wiley \& Sons, INC., New York.

9) Tsujita, M. and Tsuda, S.: The annual meeting of the Japan Electron Microscope Soc., 1953.

10) Tsujita, M. and Tsuda, S.: Ann. Rep. Nat. Inst. Genetics. 4, 51, 1953.

11) Skotland, C. B., Hagedorn, D. J. and Stahmann, M. A. : Phytopathology, 45, 603, 1955.

12) Stanley, W. M. J.: J. Biol. Chem., 126, 125, 1933.

13) Steinmann, E. and Sjöstrand, F. S.: Expt. Cell Research. 8, 15, 1955.

14) White, P. P.: Phytopathology, 24, 1003, 1934. 
Fig. Thin section of a chloxoplast within healthy tobacco leaf tissue. $\times 37,000$

Figs. 2 and 3. Thin sections of disintegrated chloroplasts within TMV-infected tobacco leaf tissue. $\times 13,000$ and $\times 6,200$

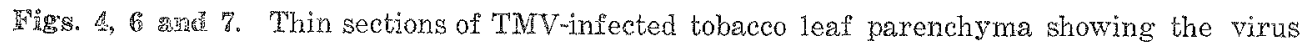
rod particles associated with chloroplasts. $\times 6,700, \times 7,700$, and $\times 9,700$

Fis. 5. Thin section of TMV rod particles surrounded with ganula from disintegrated chlorow plast. $\times 9,100$

Pig. 8. Thin section of TMV rod particles without chloroplasts. $\times 13,000$

Wig. 9. Thin section of TMV-infected epidermis. $v$ : TMV, $n$ : nucleus, $w$ : upper wall $\times 2,700$

Wig. 10. Cross section of TMV-infected tobaceo leaf epidermal hair. v: TMV, n: nucelus $\times 2,700$ 


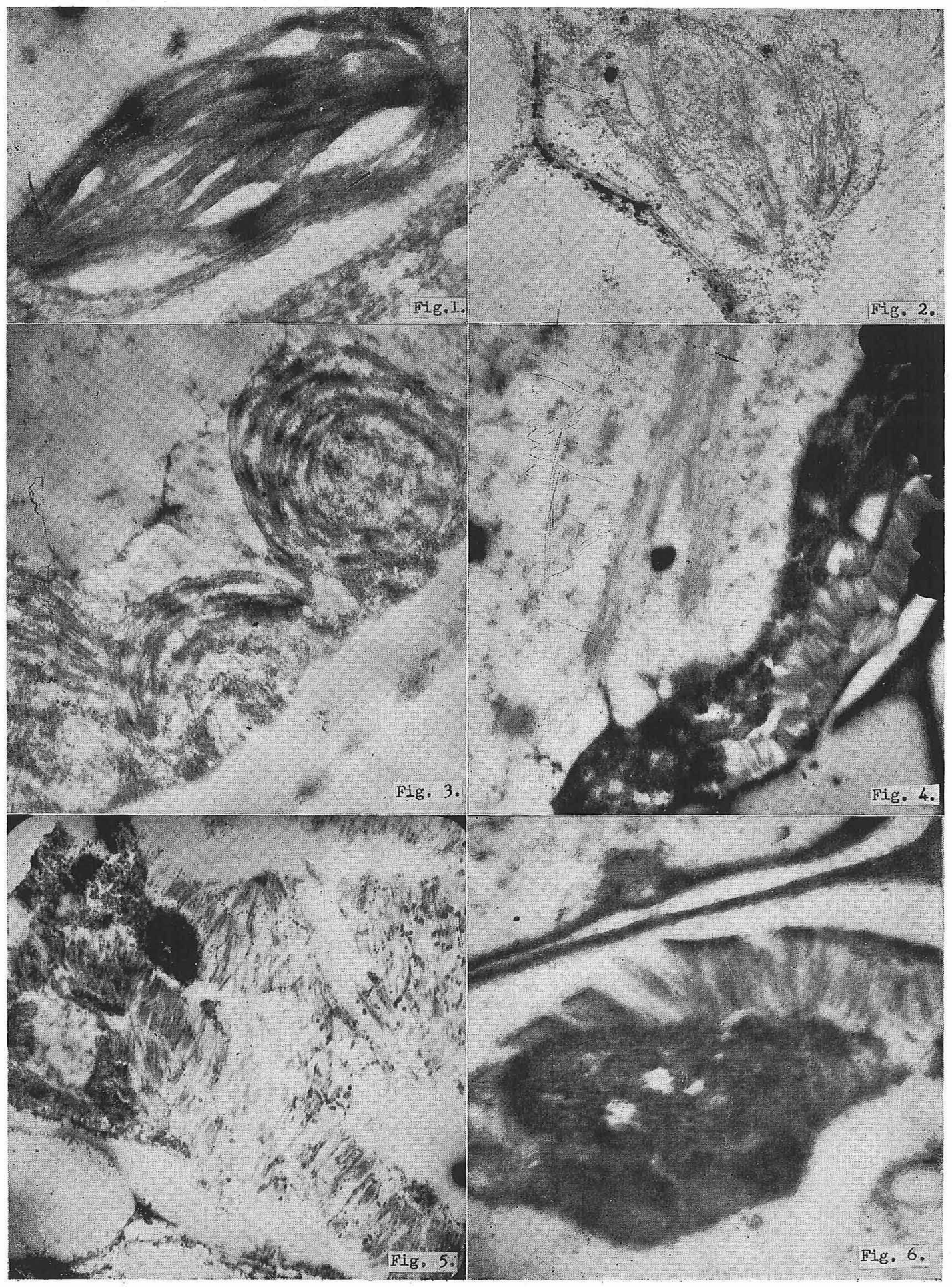



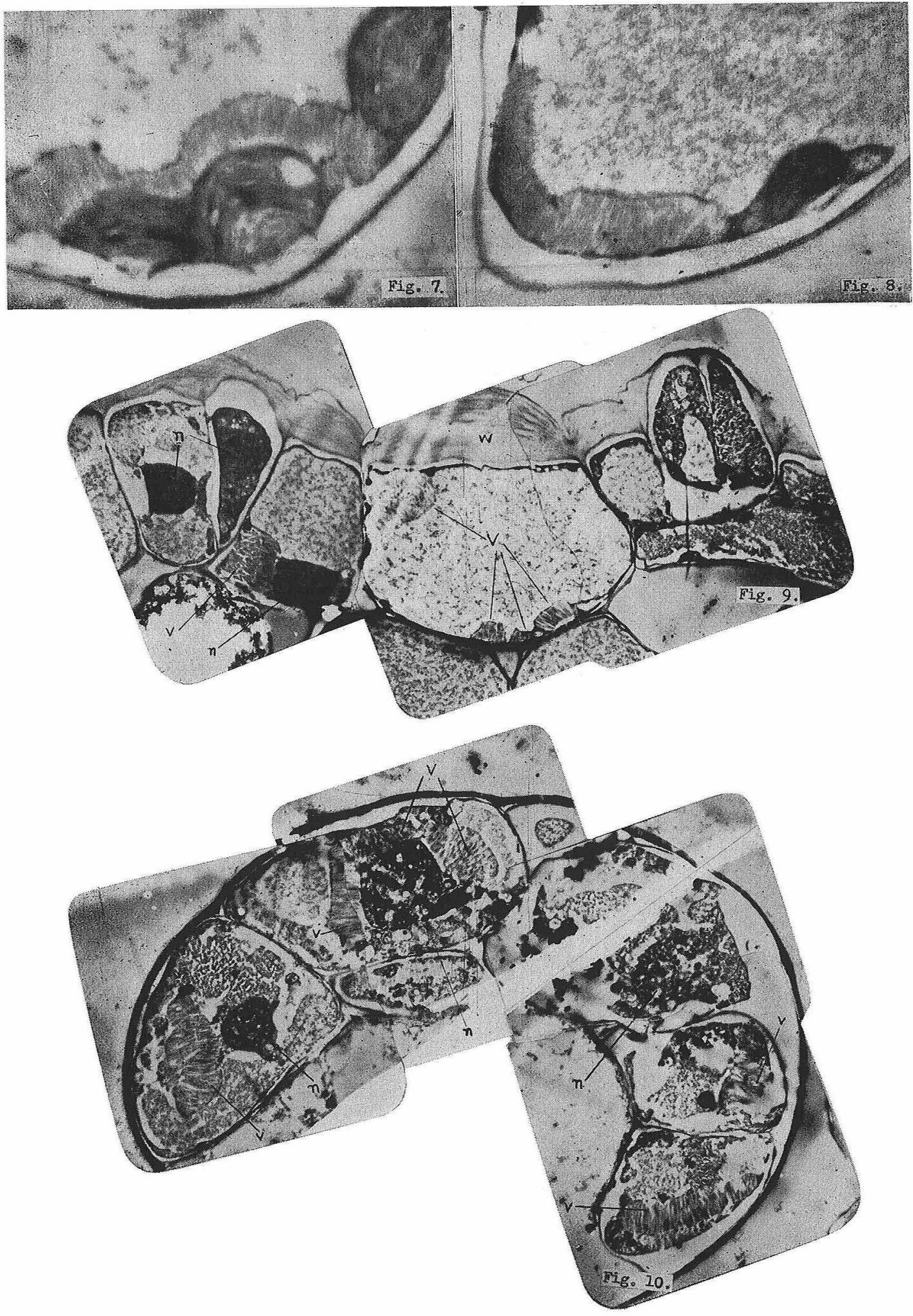